\title{
Study of the Strongly Interacting Higgs Sector
}

\author{
A. DOBADO \\ Maria J. HERRERO ${ }^{2}$ \\ CERN, CH-1211 Geneva, Switzerland \\ Tran N. TRUONG \\ CERN, CII-1211 Geneva, Switzerland \\ and
}

Centre de Physique Theorique, Fcole Polytechnique, 91128 Palaiseau, France

\begin{abstract}
The most gencral structure of the strongly interacting Higgs sector has two unknown parameters and corresponds, in this unitarized one loop calculation, to the possible existence of a $I=0, J=0$ Higgs scalar boson and a $I=1, J=1$ vector boson. The vector boson width is shown to satisfy the KSRF relation while the Higgs boson width is reduced by a fartor of $2 / 3$ from the standard tree value. As the calculation is completely unitary, no bound can be obiained for the Higgs boson and the vector boson masses.
\end{abstract}

\footnotetext{
'On leave of absence from Departamento de Fisica Teorica de la Universidad Complutense de Madrid, 28040 Madrid, Spain

${ }^{2}$ On leave of absence from Departamento de Fisica Tenrica de la Universidad Autonoma de Madrid, 28049 Madrid, Spain
}

CERN-THI.5524/89

September 1989 

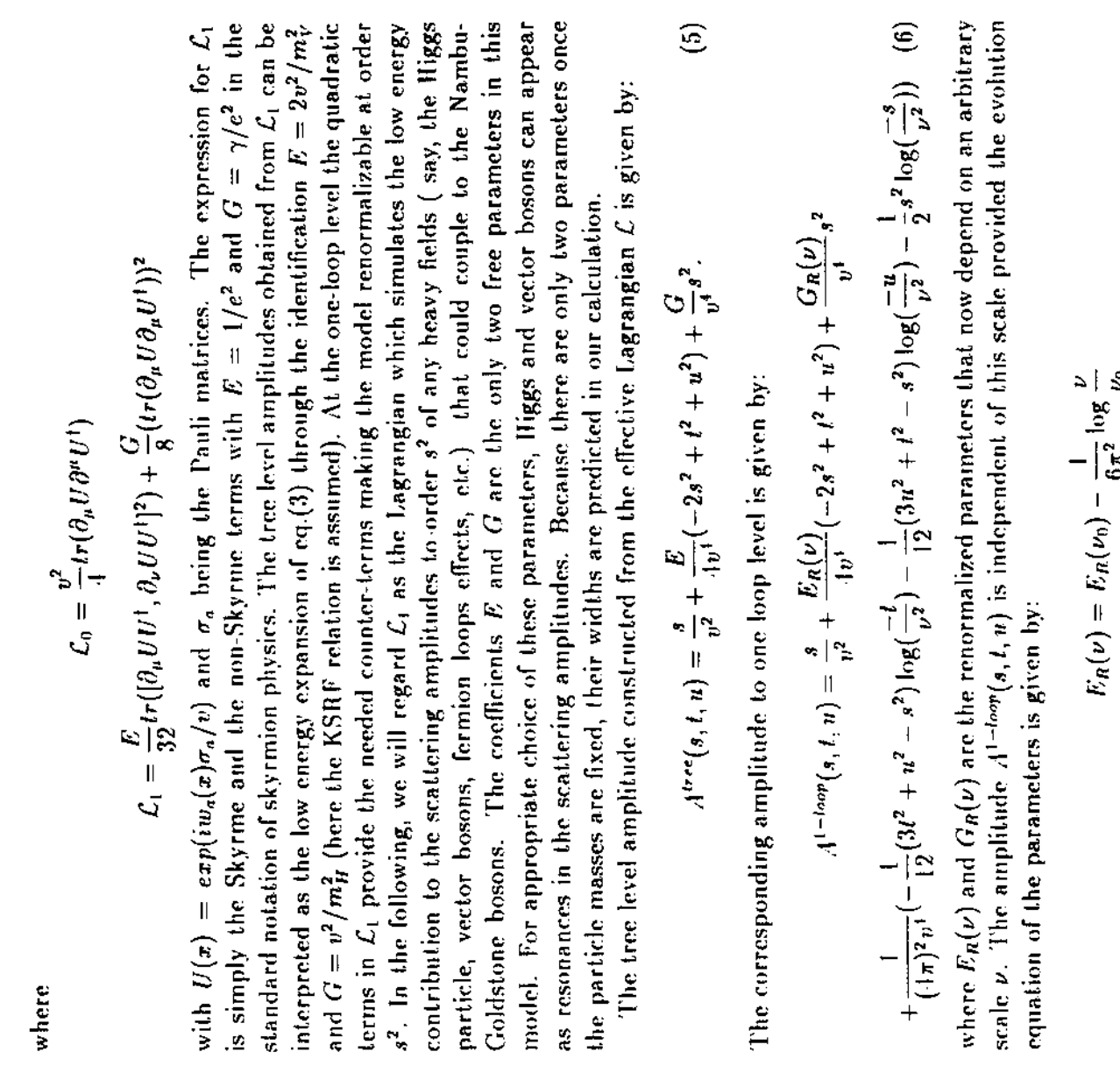
E

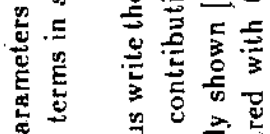

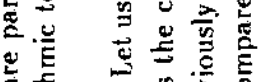

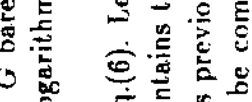

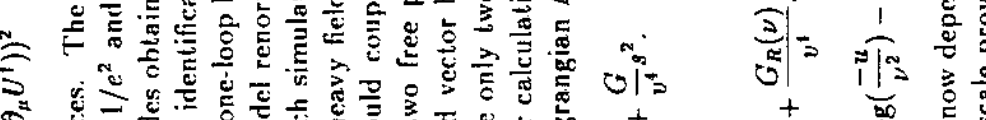

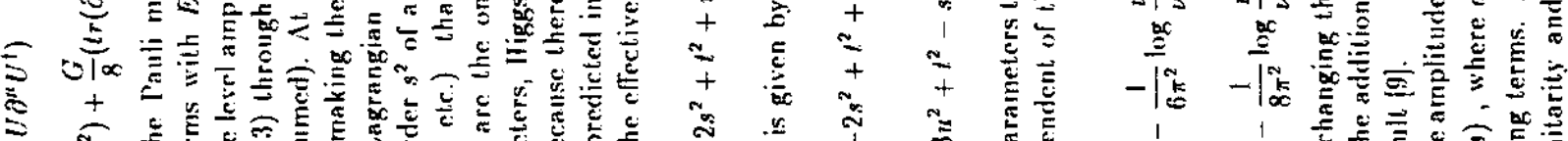

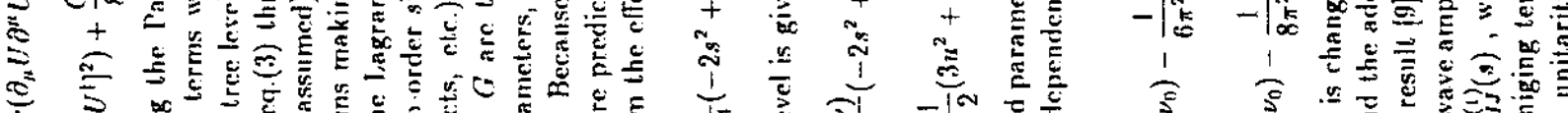

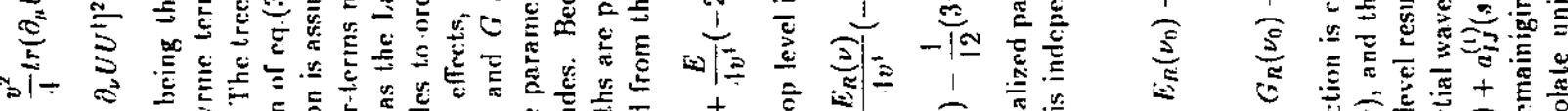

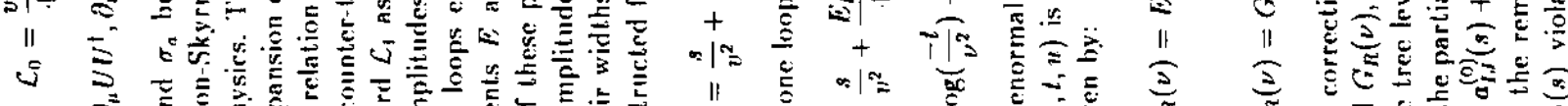

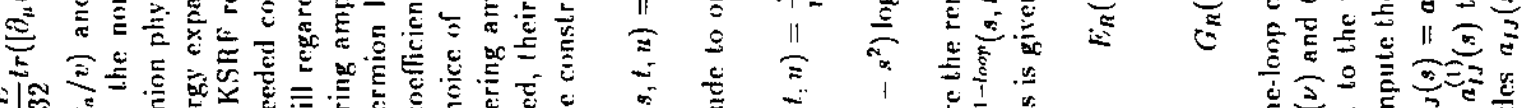

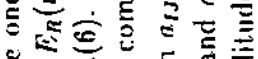

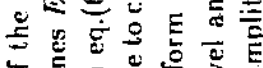

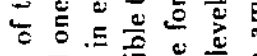

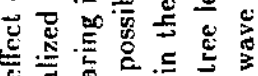

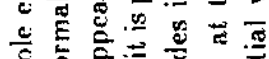

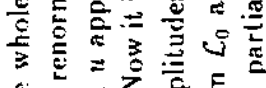

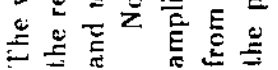

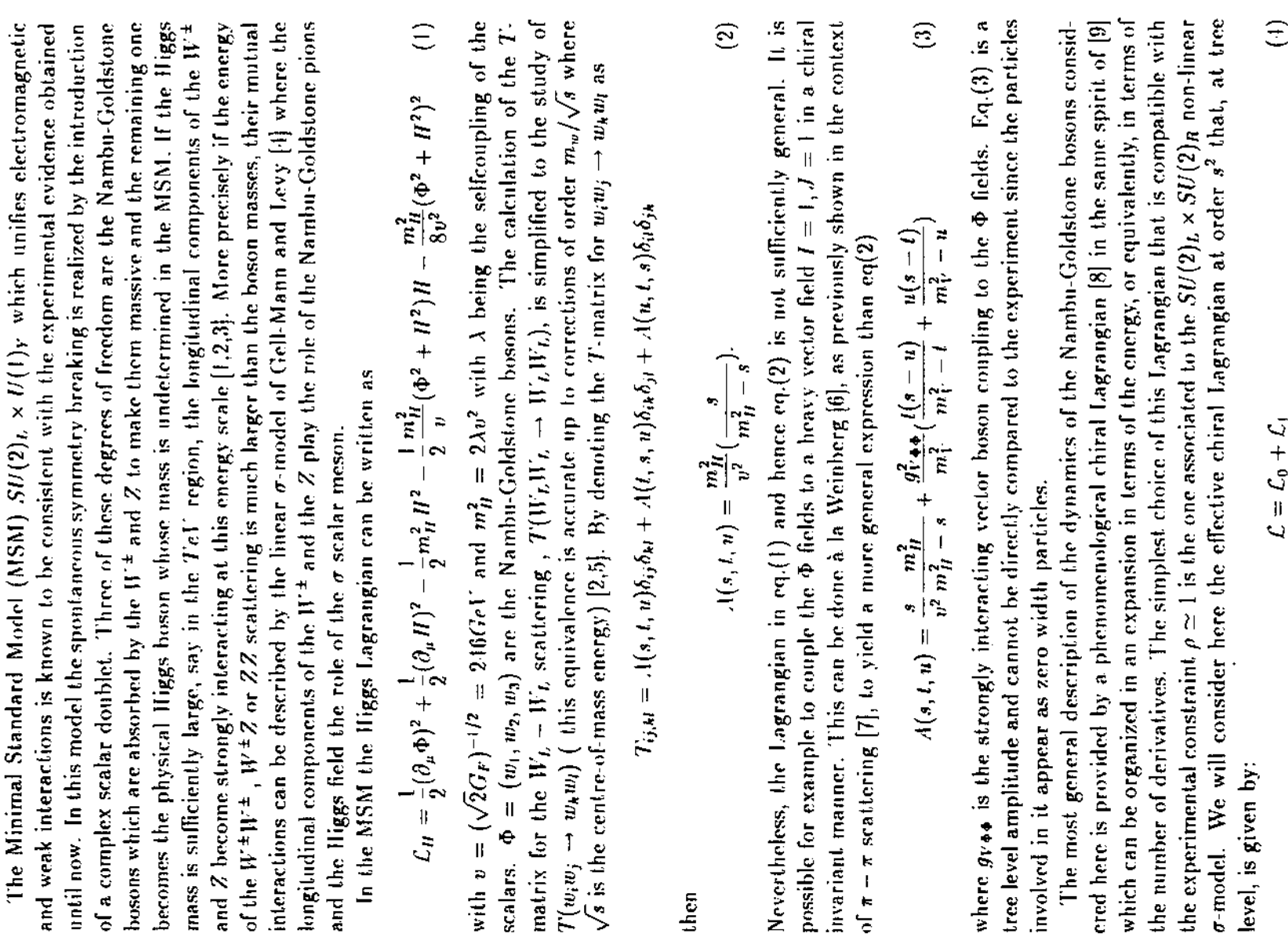



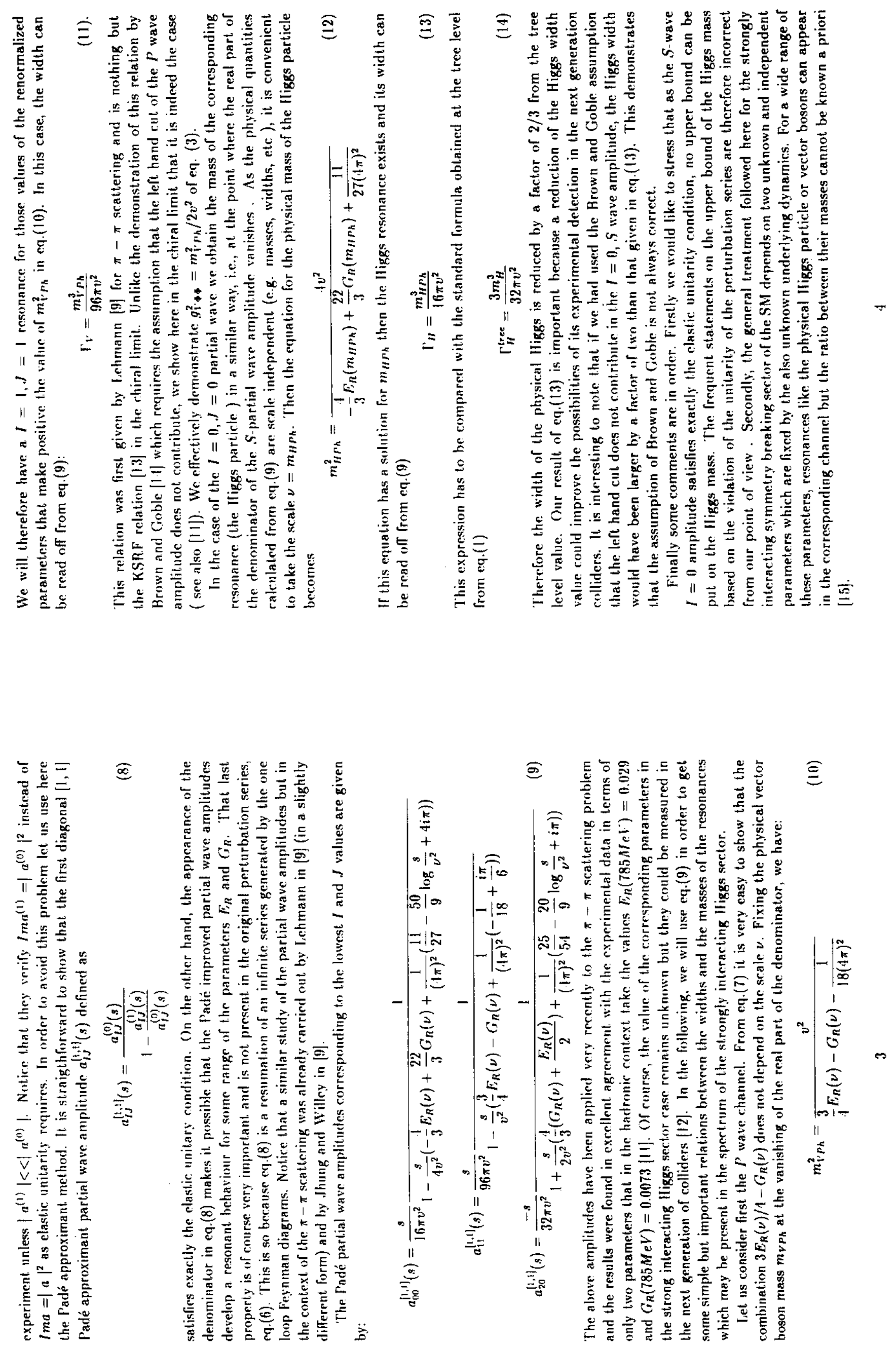

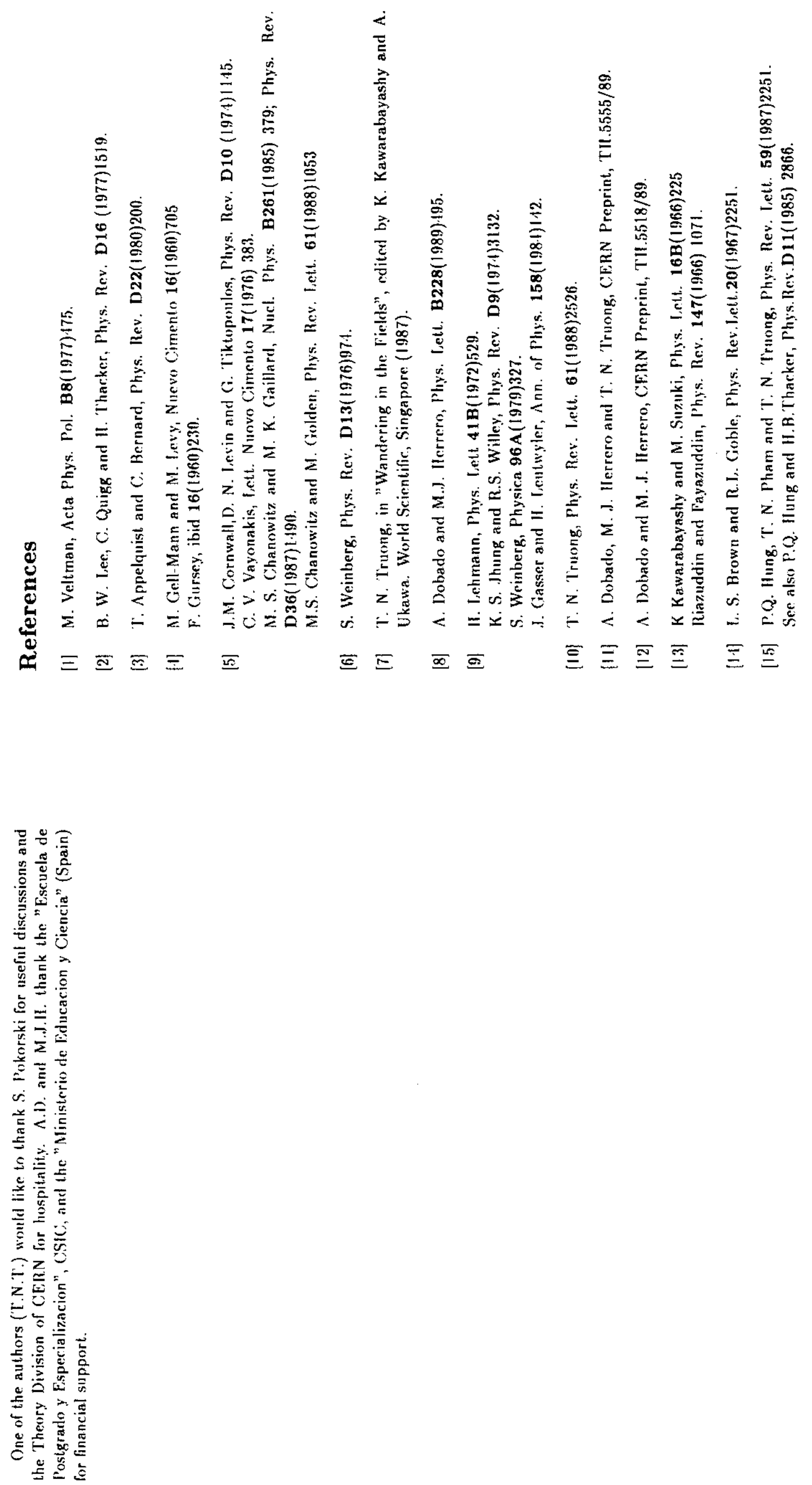